\title{
Result of using atoms and centrosymmetric cubic space groups
}

\author{
P. S. Yuen \\ 237 Des Voeux Road West, 5th Floor, Hong Kong \\ puisumyuen@netvigator.com
}

A hydrogen atom is assigned to an arbitrary general position of the space group of a centrosymmetric cubic crystal. The phases of the structure factors are obtained. These phases are associated with the $\mid \mathrm{F} \_$obs $\mid$. The result of atoms and centrosymmetric cubic space groups is that an approximate structure of the crystal is contained or embedded in the many peaks of the calculated electron densities. The purpose of this article is to establish this basic result. For applications of this property to crystal structure determination, we have some remarks:

(i) Atomic numbers and atomic scattering factors of atoms in the crystals are not employed. Hence, the electron densities reveal nonhydrogen atoms, as well as hydrogen atoms. The electron densities of the peaks do not follow the trend of the atomic numbers.

(ii) After the structures of crystals, including disordered crystals, are determined, we can compare the atomic coordinates with the peaks in the calculated electron densities, to see if there are large discrepancies, in particular, hydrogen atoms. If needed, we may apply to crystals in the literature.

(iii) Unlike Patterson peaks, the peaks in the calculated electron densities are distinct and sharp for hydrogen atoms, light atoms or heavy atoms.

(iv) Like deconvolution of Patterson peaks, we may have devices to identify the approximate structure in the calculated electron densities. Environment about each atom may be considered.

(v) If a partial structure is obtained, we may be able to locate the remaining atoms, for example hydrogen atoms, from the peaks in the calculated electron densities. The partial structure is extended. This applies to locating atoms along a polypeptide chain.

(vi) In the calculated electron densities, we may obtain peaks in special positions with coordinates fixed by symmetry. Hence we obtain a partial structure which can then be extended, as in (v).

(vii) Crystal structure may be determined by the means of a systematic and routine method [1].

(viii) This article leads to two fundamental and important questions in X-ray crystallography: What is the result of using atomic numbers and centrosymmetric cubic space groups? What is the result of using atomic scattering factors and centrosymmetric cubic space groups? If we can obtain an answer to one of these questions, the peaks in the calculated electron densities may then be classified into species of atoms. This is very useful for determination of the approximate structure.

[1] Yuen, P. S. Determination of structure of $\mathrm{CoS}_{2}$ by the means of a simple new method; a solution to the phase problem for centrosymmetric cubic crystals. (Unpublished).

Keywords: general position; equal phases; approximate structure; 